Cite this: J. Mater. Chem. A, 2013, 1, 7351

Received 18th April 2013

Accepted 9th May 2013

DOI: 10.1039/c3ta11546a

www.rsc.org/MaterialsA

\section{The room temperature formation of gold nanoparticles from the reaction of cyclohexanone and auric acid; a transition from dendritic particles to compact shapes and nanoplates $\dagger$}

\author{
Madeeha A. Uppal, ${ }^{\text {a }}$ Andreas Kafizas, ${ }^{\text {b }}$ Michael B. Ewing ${ }^{a}$ and Ivan P. Parkin*a \\ A new straightforward method for the synthesis of gold nanoparticles from addition of cyclohexanone to \\ aqueous solutions of auric acid at room temperature is presented. By understanding this process we have \\ discovered a new organic chemistry transformation reaction for converting cyclic ketones to $\alpha$-chloro \\ ketones and a mechanism for the nanoparticle formation. Contrary to conventional gold nanoparticle \\ syntheses, the reaction "self-initiates" at room temperature and forms an increasingly red solution over \\ $\approx 60$ minutes. By studying the gold colloid's formation using transmission electron microscopy it was \\ observed that large dendritic ( $63 \pm 21 \mathrm{~nm}$ diameter) structures made of clustered particles (6 $\pm 1 \mathrm{~nm}$ ) \\ were initially formed. These dendritic particles then compacted into an array of denser shapes that slowly \\ increase in size until the reaction is complete. The most prominent shapes observed were spheres $(43 \pm 7$ \\ $\mathrm{nm})$; other shapes included dodecahedra $(39 \pm 10 \mathrm{~nm})$ triangular $(\approx 50 \mathrm{~nm}$ in height) and hexagonal \\ ( $\approx 70 \mathrm{~nm}$ wide) nanoplates. The solution was stable to precipitation for over 3 months. During this \\ period the nanoplate structures substantially increased in size (triangular $\approx 250 \mathrm{~nm}$, hexagonal $\approx 320$ \\ $\mathrm{nm}$ ) whereas other structures showed no further growth. X-ray diffraction studies demonstrated that the \\ gold nanoparticles were crystalline. The formation of the 2-chlorocyclohexanone by-product was \\ observed in solution phase ${ }^{1} \mathrm{H} \&{ }^{13} \mathrm{C} N M R$, gas phase chromatography and IR spectroscopy. A mechanism \\ is presented to account for this by-product and the reduction of auric acid to gold.
}

\section{Introduction}

Gold nanoparticle colloids have received a great deal of interest because of their intense and size/shape tuneable surface plasmon resonance (SPR) properties..$^{1-3}$ Gold nanoparticles are used in a wide sector of applications as optical enhancers in composite semiconductor films, ${ }^{\mathbf{4 - 7}}$ bactericides, ${ }^{\mathbf{8 , 9}}$ biological markers, ${ }^{\mathbf{1 0}}$ DNA and single molecule detectors ${ }^{\mathbf{1 1}, \mathbf{1 2}}$ and in catalysts. ${ }^{13}$ The most common method for preparing gold colloids is based on the thermal reduction of aqueous auric acid with tricitrate, devised by Turkevich et al.; ${ }^{\mathbf{1 4 1 5}}$ where solutions are typically heated to $100{ }^{\circ} \mathrm{C}$ for the reaction to initiate. Gold nanoparticles have been synthesized by heating auric acid solutions with a variety of fruit extracts. ${ }^{16}$ These fruit extracts contained several ketone functional groups and it may be that these functional groups mediate the formation of multi-shaped nanoparticles. Such multi-shaped gold nanoparticles have been

${ }^{a}$ Department of Chemistry, University College London, 20 Gordon Street, London, WC1H 0AJ, UK. E-mail: i.p.parkin@ucl.ac.uk; Fax: +44 (0)20 76797463

${ }^{b}$ Department of Chemistry, Imperial College London, South Kensington Campus, London $S W 72 A Z$, UK

$\dagger$ Electronic supplementary information (ESI) available: Zoomed-in ${ }^{13} \mathrm{C}$ NMR spectra identify by-products in the reaction. See DOI: $10.1039 / \mathrm{c} 3 \mathrm{ta11546a}$ used to investigate the biological toxicity of gold nanoparticles in zebrafish embryos ${ }^{17}$ and mammalian cells ${ }^{18}$ but are yet to be applied in a functional material. Silver nanoparticles of different shapes and sizes have also been found to induce multicolour photochromism when photo-chemically loaded onto $\mathrm{TiO}_{2}$ thin-films. ${ }^{19}$

The use of ketones in precious metal nanoparticle synthesis has been reported - although all routes involve significant heating and no mechanism for their formation has been proposed. Truncated gold nanocubes could be produced using an electrochemical approach and an acetone injection stage. ${ }^{20,21}$ Dendritic nanoparticle structures comprised of Pd have been synthesized by reaction of the Pd acetate salt mixed with ethylene glycol and $\mathrm{KOH}$ in a methyl isobutyl ketone solvent initiated by microwave heating. ${ }^{22}$ This formed stable $\approx 50 \mathrm{~nm}$ wide dendritic structures. The formation of large $2 \mathrm{D}$ triangular and hexagonal nanoplates has also been previously observed..$^{23-25}$ By mixing gold seeds with a mixture of CTAB, PEG and the ketone polymer PVP a slow room temperature reaction over 8 hours produced platelet-type particles that were typically $\approx 5 \mathrm{~nm}$ thick and $\approx 200$ to $500 \mathrm{~nm}$ wide. It was also shown that microwave heating $\left(85{ }^{\circ} \mathrm{C}\right)$ of aqueous solutions of auric acid with long-chained PVPs yielded similar structures. ${ }^{26}$ Mixing 
aqueous auric acid solutions with a lemongrass plant extract (Cymbopogon flexuosus) at room temperature produced nanoplate mixtures with lengths on the micrometer scale, where the directional growth was related largely to the ketone groups present in the extract. $^{27}$

To our knowledge, the use of cyclohexanone in initiating a self-propagating gold nanoparticle formation reaction has not previously been reported. Nevertheless, the use cyclohexanonewater-isopropanol mixtures in synthesizing a range of copper based nanoparticles ${ }^{28}$ and cyclohexanone as solvent and capping agent for forming gold nanoparticles through laser ablation of a submerged gold target. ${ }^{29}$ It was also shown that aryl ketones can photocatalytically reduce solutions containing $\mathrm{Au}^{3+}$ to $\mathrm{Au}^{0} \cdot{ }^{30}$ The growth mechanisms for traditional gold nanoparticle formation methods have come to be somewhat understood through in situ monitoring methods such as UV-visible spectroscopy, dynamic light scattering, electrode potential measurements or through the extraction and transition electron microscopy analysis of aliquots at set times during the reaction. ${ }^{31,32}$

We show that cyclohexanone reacts with auric acid and causes a "self-initiated" room temperature nanoparticle formation reaction to commence. During this reaction the particles undergo several shape changes. Initially, large dendritic structures composed of smaller aggregates form that later compact primarily into spherical particles and a fraction form alternative 3D structures such as triangular prisms and dodecahedrons and large flat 2D hexagonal and triangular nanoplates. We also provide an insight into the underpinning mechanism showing that the cyclohexanone acts as the reducing agent; reducing $\mathrm{Au}^{3+}$ in auric acid to $\mathrm{Au}^{1+}$ (that later disproportionates to $\mathrm{Au}^{0}$ ) and being converted to 2-chlorocyclohexanone in this process. This new method of multi-shaped nanoparticle synthesis is reproducible and amongst the simplest reported ${ }^{33,34}$ in that it involves no heating stage, occurs at room temperature and just requires one stage of reagent mixing. Furthermore, the nanoparticles formed are stable against precipitation for more than 3 months stored at room temperature.

\section{Experimental section}

All reagents were purchased from Sigma-Aldrich UK. All glassware was washed with aqua regia and rinsed with copious amounts of de-ionized water before use. All reactions were performed in beakers.

The nanoparticle solution was made by mixing auric acid $\left(\mathrm{HAuCl}_{4} \cdot 3 \mathrm{H}_{2} \mathrm{O}-0.85 \mathrm{mg}, 0.28 \mathrm{mM}\right)$ in distilled water $(9.5 \mathrm{ml})$ with cyclohexanone $\left(\left(\mathrm{CH}_{2}\right)_{5} \mathrm{CO}-0.50 \mathrm{ml}, 0.47 \mathrm{~g}, 0.48 \mathrm{M}\right)$. This solution did not require thermal activation (or any alternative energy source) and "self-initiated" the uniform formation of gold nanoparticles throughout the solution when conducted under room light or in a dark cupboard. The reaction was accompanied by a darkening of the solution. A further experiment was conducted where the cyclohexanone was injected gently on top of the aqueous auric acid solution to form a separate organic layer on top of the aqueous. In this case, the nanoparticle formation reaction occurred only at the emulsion interface as judged by the change in colour. The effect of temperature on the reaction was investigated by mixing auric acid $(2.6 \mathrm{mg}, 0.28 \mathrm{mM}$ ) in distilled water $(28.5 \mathrm{ml})$ with cyclohexanone (1.5 ml, $1.4 \mathrm{~g}, 0.48 \mathrm{M})$ at $\approx 20,30,40,50$ and $60{ }^{\circ} \mathrm{C}$. After first heating the aqueous auric acid solution to the desired temperature, the cyclohexanone reducing agent was rapidly injected, causing a thorough mixing of the solutions. The rate of consumption of auric acid in the reaction was determined by titrating solution aliquots during the nanoparticle formation reaction. ${ }^{35}$ This was achieved by scaling the reaction up by a factor of 5 and taking $2 \mathrm{ml}$ aliquots out every 5 minutes for analysis. The nanoparticle formation reaction was quenched as soon as the aliquots were extracted by addition of excess KI $(0.02 \mathrm{~g})$. This caused all auric acid present within the aliquot to be consumed to AuI, forming an $\mathrm{I}_{2}$ side-product. The level of $\mathrm{I}_{2}$ formed, and thus the amount of auric acid originally present, was measured by titration against $\mathrm{K}_{2} \mathrm{~S}_{2} \mathrm{O}_{3}(1 \mathrm{mM})$ using potato starch indicator $(0.02 \mathrm{~g})$ where the disappearance of blue-black colour indicated completion.

The size and shape of nanoparticles formed at room temperature by rapid mixing of the solution were investigated using transmission electron microscopy (TEM) using a Jeol 4000EX microscope. Solution aliquots were taken out from the nanoparticle reaction after 5, 20 and 65 minutes of reaction and placed onto lacey carbon-coated copper grids and allowed to air dry before being investigated by TEM.

Nanoparticles solutions formed at room temperature by rapid mixing were freeze dried to remove the aqueous solvent and any excess cyclohexanone to form a nanoparticle powder. The powder was analyzed by X-ray diffraction (XRD) using a micro-focus Bruker GADDS powder X-ray diffractometer with a monochromated $\mathrm{CuK}_{\alpha}(1.5406 \AA)$ source and CCD area detector. After mixing the powder within a pressed $\mathrm{KBr}$ disc, the infrared (IR) spectrum of the solid was taken using a PerkinElmer RX-I instrument.

Nanoparticle solutions were centrifuged on a Beckman Coulter Avanti Centrifuge J-26 XP at $20000 \mathrm{rpm}(\approx 48000 g)$ causing the gold particles to rest at the bottom of the solution. The liquid layer above was removed and passed through a gas chromatograph (Clarus 500, PerkinElmer) equipped with flame ionization detector and $30 \mathrm{~m}$ capillary column (Ellite-1, crossbond $100 \%$ dimethyl polysiloxane) and compared with standards to elucidate the reaction by-products. The gases released from the nanoparticle reaction were analyzed by IR spectroscopy and compared with the pure gas phase spectra of cyclohexanone and 2-chlorocyclohexanone in a sealed gas cell under vacuum. Three samples were examined by both ${ }^{1} \mathrm{H}$ and ${ }^{13} \mathrm{C}$ nuclear magnetic resonance (NMR) spectroscopy using a Bruker AV600 (600 MHz) instrument: (i) a gold nanoparticle solution (diluted by a factor of 100) formed from the reaction of auric acid and cyclohexanone in $\mathrm{D}_{2} \mathrm{O}$ at room temperature, (ii) cyclohexanone in $\mathrm{D}_{2} \mathrm{O}$ and (iii) 2-chlorocyclohexanone in $\mathrm{D}_{2} \mathrm{O}$.

\section{Results and discussion}

The self-initiated formation of gold nanoparticles was induced by reacting a thousand fold excess of cyclohexanone with aqueous solutions of auric acid at room temperature. Several 
photographs were taken at set time intervals during the selfinitiated nanoparticle formation reaction, as displayed in Fig. 1. Two reactions were studied; (i) the rapid mixing of cyclohexanone with auric acid and (ii) the layering of cyclohexanone on top of the aqueous auric acid layer. In the case of thorough premixing of the cyclohexanone reducing agent with the auric acid solution (Fig. 1(a)) no colour change was observed until after $\approx 10$ minutes when the solution turned slightly pink. This coloration became more intense as the reaction proceeded. After $\approx 40$ minutes of reaction the solution appeared purple and was more strongly coloured. After $\approx 60$ minutes of reaction, the solution did not further deepen in colour, signifying the end of the gold nanoparticle formation reaction. When the solution was viewed off-angle, a two-tone effect was revealed where the solution showed a more orange tone when viewed at high angles and a more purple tone when looked at directly.

In the case of gentle layering of cyclohexanone at the surface of the aqueous auric acid solution, an emulsion layer was formed in which the colour change and hence nanoparticles formation occurred solely at the cyclohexanone-water interface. Fig. 1(b) shows that just after addition of the reducing agent (0 min), two separate immiscible layers can be observed. Approximately 20 minutes into the reaction a pink layer began to form within the aqueous layer at the interface. As the reaction progressed, the pink layer deepened in colour and penetrated deeper into the aqueous layer as the nanoparticles diffused. After 60 minutes of reaction, almost half of the aqueous layer had turned pink. On comparing the two reactions it is clear that nanoparticle formation is more rapid in the solution that was thoroughly pre-mixed. However, one can also infer the importance of the partial miscibility of the cyclohexanone on the mechanism of nanoparticle formation.

The nanoparticle formation reaction, where the cyclohexanone initiator was well-mixed, was also followed using UVvisible spectroscopy over the 190-1100 nm range, as shown in Fig. 2. The spectra showed the formation of a band at 540$575 \mathrm{~nm}$ corresponding to the surface plasmon resonance (SPR)
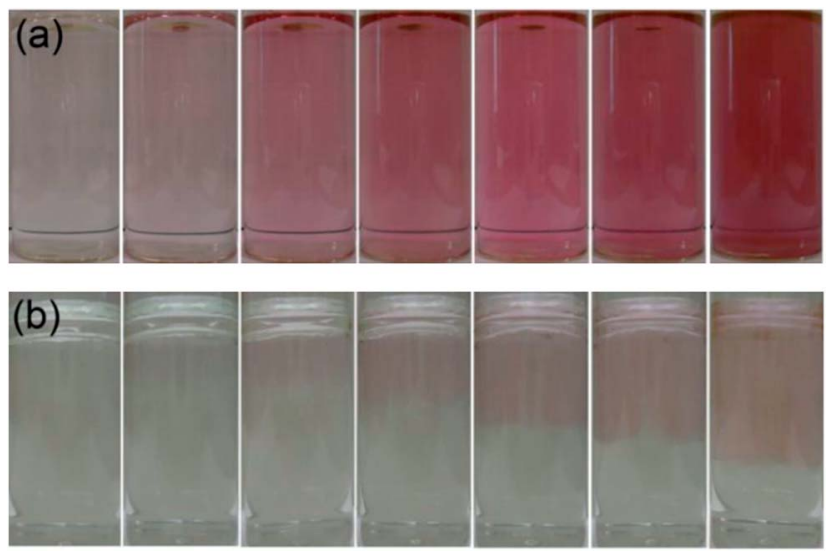

Fig. 1 A series of images of the same solution taken at time intervals of $0,10,20$, $30,40,50$ and 60 minutes reaction time (from left to right) in the self-initiated gold nanoparticle formation reaction of auric acid solution with cyclohexanone that was (a) shaken into the solution or (b) layered gently on top of the aqueous layer.

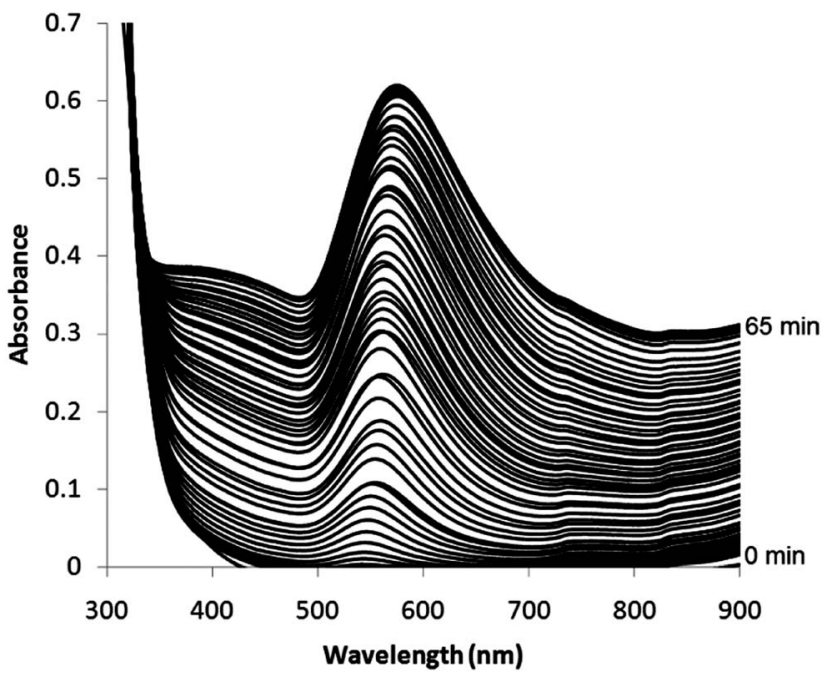

Fig. 2 UV-visible spectrum from 300 to $900 \mathrm{~nm}$ monitoring the growth of gold nanoparticles in situ during their self-initiated formation reaction from an aqueous auric acid solution and cyclohexanone. Each spectrum was taken approximately every 70 seconds and the plasmon band appeared $\approx 10$ minutes after the addition of cyclohexanone. The spectra are shown as recorded and no shift was applied to the baseline - this naturally shifted upwards with the growth of the SPR band.

of Au nanoparticles. The UV-visible spectrum was taken every $\approx 70$ seconds until the SPR band stopped increasing. Approximately 10 minutes after the cyclohexanone was added a SPR band started to appear. This continued to increase at a relative steady rate until after $\approx 65$ minutes after cyclohexanone addition, when the SPR band suddenly stopped increasing in magnitude. The overall baseline also increased its absorbance during the process - this stopped its upward shift at the same time as the SPR band showed no further increase in magnitude.

The centre (nm) and height (absorbance) of each progressively growing SPR band was extracted and plotted against reaction time (minutes), as shown in Fig. 3. As was seen in UVvisible absorbance spectra, an SPR band (543 nm, 0.01 absorbance units) first appeared 10 minutes after the addition of cyclohexanone. The SPR band then grew in height at a relatively steady rate of roughly 0.012 absorbance units per minute. The centre of the SPR also shifted to longer wavelengths. These two properties continued to increase until exactly 64 minutes after the addition of cyclohexanone, where they both reached a plateau. From 64 to 75 minutes reaction time both the SPR centre and height did not change significantly. Over the reaction period the SPR height increased from 0 to 0.61 absorbance units. In addition, the SPR centre shifted from 543 to $576 \mathrm{~nm}$. The solutions were highly stable to precipitation and altered little after being stored for 3 months; although the (SPR centre decreased to $563 \mathrm{~nm}$ and the absorbance dropped to 0.59).

It is well known that an increase in SPR centre wavelength signifies an increase in average nanoparticle size. ${ }^{15}$ Using the relationship derived by Haiss et al. ${ }^{36}$ the increase in SPR centre from 543 to $576 \mathrm{~nm}$ signifies an average increase in nanoparticle diameter of $\approx 34 \mathrm{~nm}$, assuming the particles are predominantly spherical. 


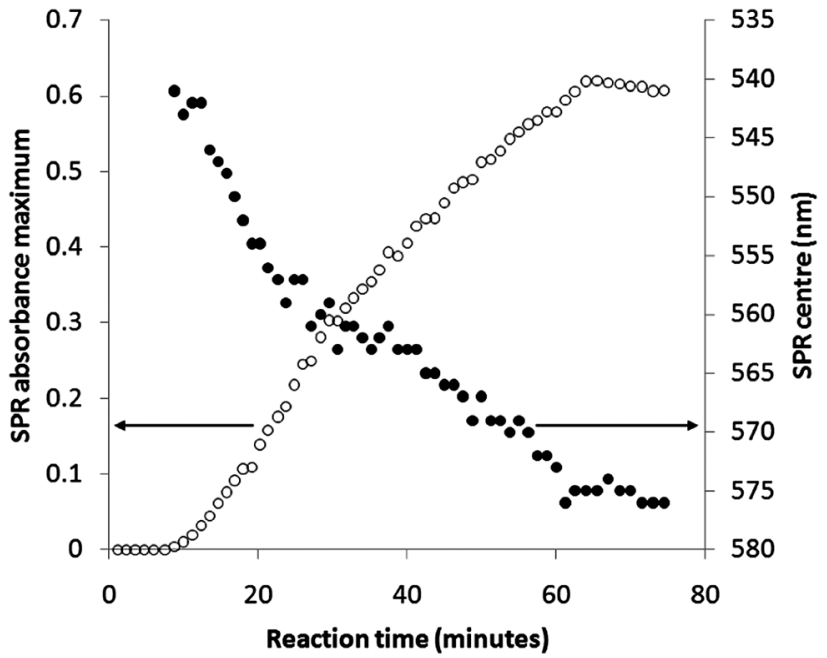

Fig. 3 A plot of the centre $(\mathrm{nm})$ and height (absorbance) of the progressively growing surface plasmon resonance band extracted from UV-visible spectra monitoring the growth of gold nanoparticles in situ during their self-initiated formation reaction from auric acid solution and cyclohexanone.

The rate of consumption of auric acid in the reaction was determined by titrating solution aliquots during the nanoparticle formation reaction. ${ }^{35}$ The level of auric acid was found to decrease linearly over the time period (Fig. 4); decreasing from $0.26 \mathrm{mM}$ at the start of the reaction ( 0 minutes) at a rate of $6.2 \mu \mathrm{M} \mathrm{m^{-1 }}$ to there being no presence of auric acid after 45 minutes of reaction. This was in contrast with nanoparticles formed by the reaction of auric acid and citric acid (the Turkevich method), where during the reaction a sharp change in auric acid concentration is observed at a critical point. ${ }^{31}$ From our spectroscopic studies it was observed that surface plasmon

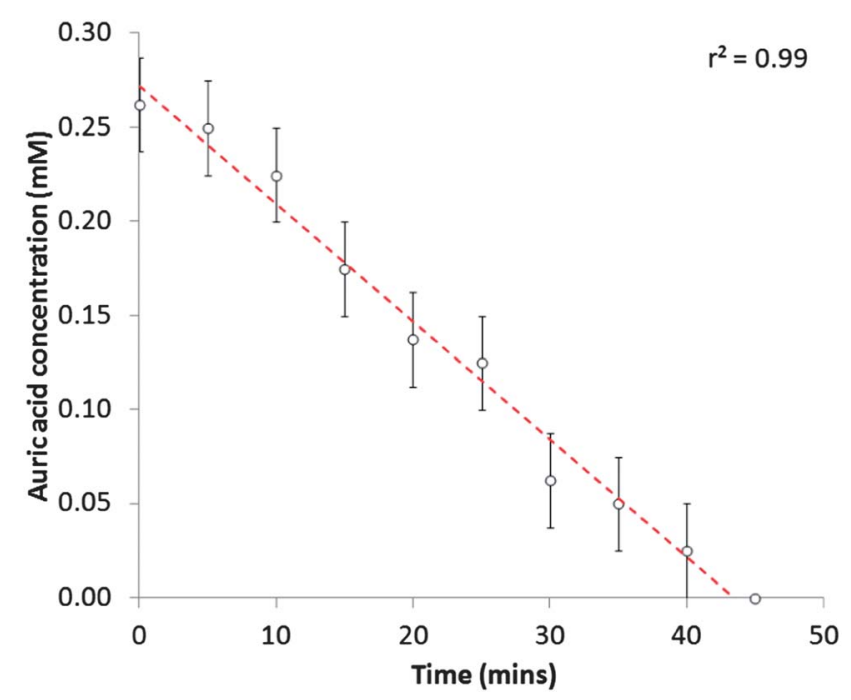

Fig. 4 Auric acid ( $\mathrm{mM}$ ) depletion in the self-initiated gold nanoparticle formation reaction of auric acid $(0.28 \mathrm{mM})$ with cyclohexanone $(0.48 \mathrm{M})$. This was measured by quenching the reaction in sample aliquots with iodide salt and measuring the level of $\mathrm{I}_{2}$ side-product by titration with thiosulfate using starch indicator. The decrease in auric acid concentration was strongly linear $\left(r^{2}=0.99\right)$. band formation, indicating the formation of gold nanoparticles, terminated after $\approx 65$ minutes of reaction, whereas our titrations showed that the auric acid was depleted after 45 minutes. This showed that there was a 20-minute lag between the complete reduction of gold ions and their subsequent nucleation to form nanoparticles with detectable surface plasmon resonance band.

The gold nanoparticle formation reaction was repeated with solution aliquots being extracted 5, 20 and 65 minutes after the addition of cyclohexanone and placed on lacey carbon-coated copper grids for transmission electron microscopy (TEM) analysis. Representative TEM images are shown in Fig. 5. Fig. 5(a) shows that after 5 minutes of reaction dendritic structures had formed, which ranged in size from 20 to $93 \mathrm{~nm}$ in diameter. The average diameter and standard deviation of these dendritic structures was $63 \pm 21 \mathrm{~nm}$. On closer inspection it can be seen that these dendritic structures are composed of clusters of smaller particles. These particles were predominantly spherical and ranged in size from 4.0 to $10 \mathrm{~nm}$. The average diameter and standard deviation of these particles of which the dendritic structures were composed was $6 \pm 1 \mathrm{~nm}$. Fig. 5(b) shows a less magnified image of the same sample. Some wellformed gold nanoparticles had formed alongside the dendritic structures. Mainly spherical shapes $(11 \pm 7 \mathrm{~nm})$ were observed, however dodecahedral $(21 \pm 2 \mathrm{~nm})$ and rod-like $(33 \pm 17 \mathrm{~nm}$ in length) gold nanoparticle shapes were also present. Intriguingly, large nanoplates (flat structures) were also observed that were mainly triangular in shape ( $29 \pm 12 \mathrm{~nm}$ in height). Fig. 5(c) shows that after 20 minutes of reaction these large dendritic structures had disappeared and only more compact looking and more regularly shaped gold nanoparticles were present. Spherical nanoparticles were still the most abundant shape, but had more than doubled in average size from $11 \pm 7 \mathrm{~nm}$ after 5 minutes of reaction to $25 \pm 7 \mathrm{~nm}$ after 20 minutes of reaction. Dodecahedral ( $28 \pm 5 \mathrm{~nm}$ ), rod-like ( $49 \pm 23 \mathrm{~nm}$ in length) and triangular nanoplate $(29 \pm 5 \mathrm{~nm}$ in height) structures were also still present. However, some extra shapes, not previously observed after 5 minutes of reaction were now observed, such as decahedra (31 $\pm 4 \mathrm{~nm})$, trigonal bipyramids (35 $\pm 6 \mathrm{~nm})$ and triangular prisms $(30 \pm 5 \mathrm{~nm})$. Fig. $5(\mathrm{~d})$ shows the final state of the nanoparticles after they had been allowed to react to completion (65 minutes total reaction time). No new shapes were observed and all of the various kinds of nanostructure observed after 20 minutes of reaction were still present, except for triangular prisms. The diameter/length of each type of shape had increased on average by $\approx 20 \mathrm{~nm}$. Spheres were still the most predominant shape, increasing in size from $25 \pm 6.4 \mathrm{~nm}$ after 20 minutes of reaction to $43 \pm 7 \mathrm{~nm}$ after 65 minutes of reaction. Colloidal solutions were stable to precipitation upon prolonged storage (at least 3 months). A TEM image size analysis of such a solution after 3 months of storage demonstrated how little the predominantly spherical nanoparticles changed in size $(50 \pm 10 \mathrm{~nm})$. However, the primarily flat nanoplate structures showed an almost 5-fold increased in size (Fig. 5(e) and (f)). These nanoplates were similar in size and shape to those formed by Tsuji et al. from the microwave heating $\left(85^{\circ} \mathrm{C}\right)$ of aqueous solutions of auric acid with long-chained PVPs. ${ }^{26}$ 
(a)
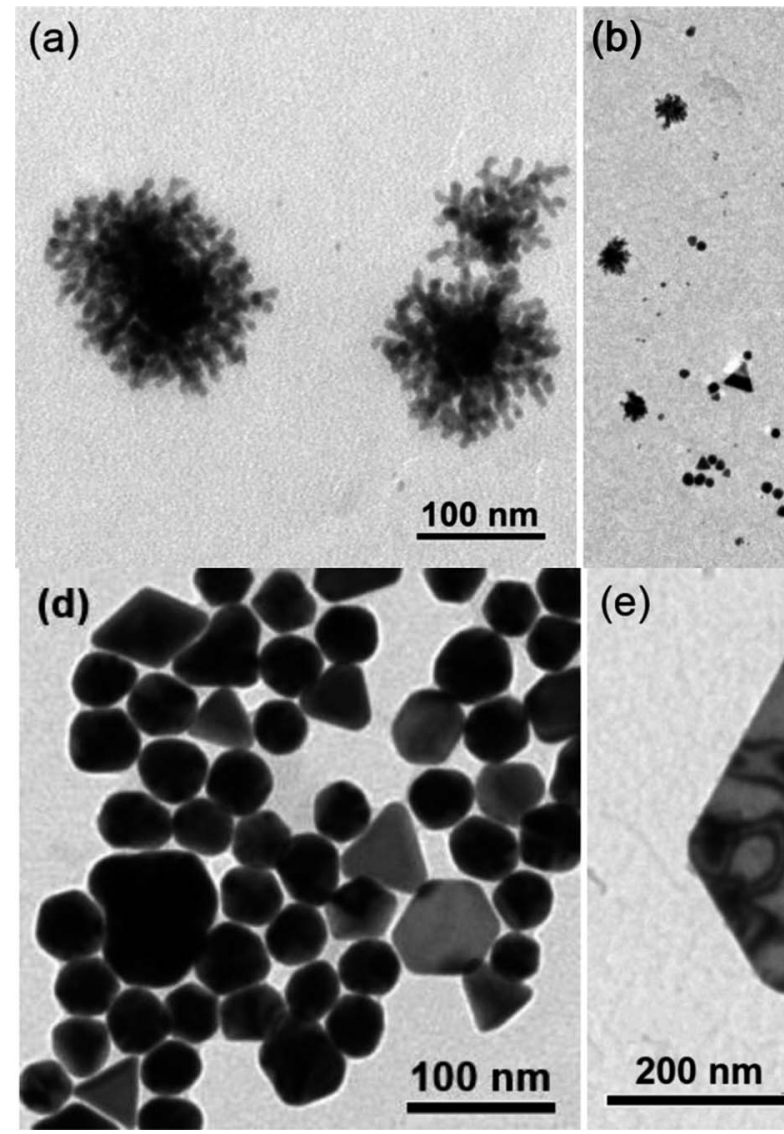

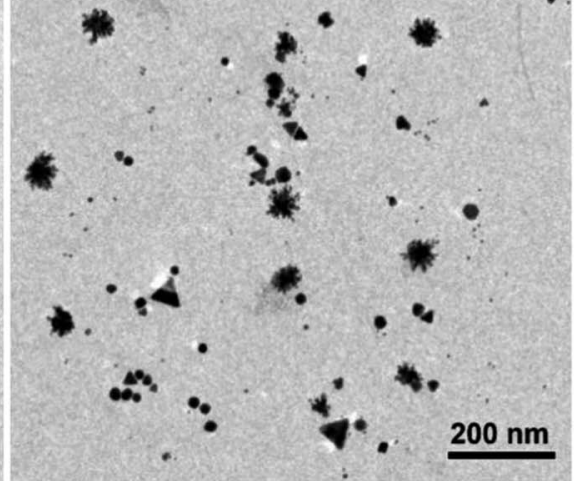

(e)

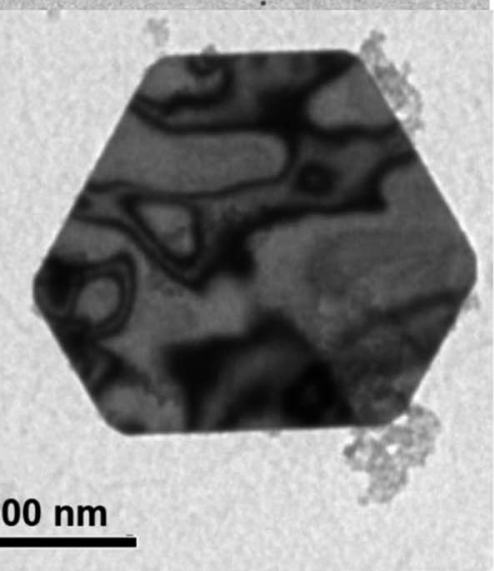

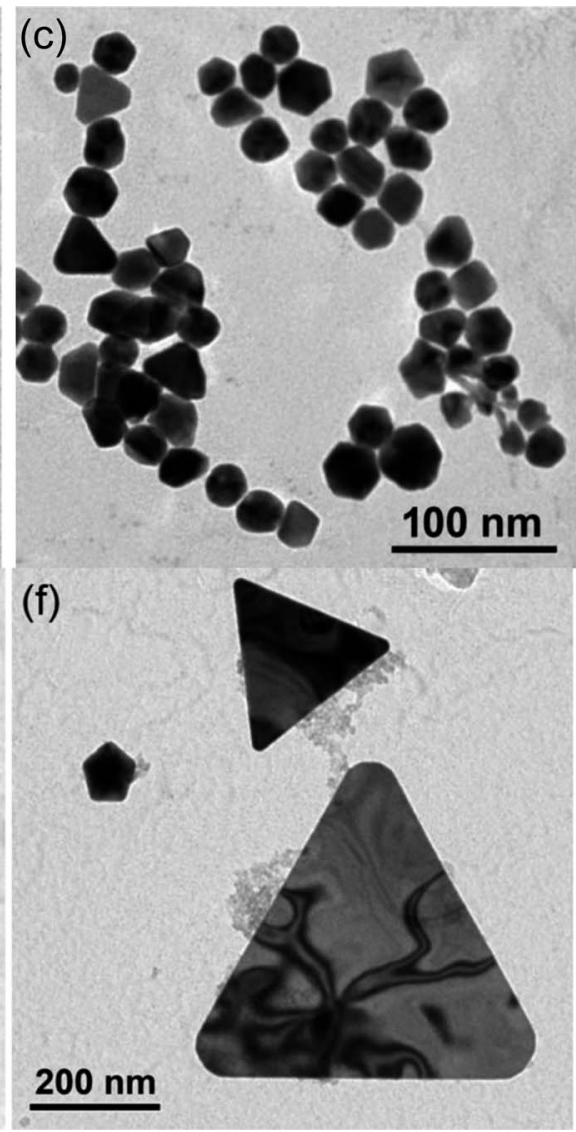

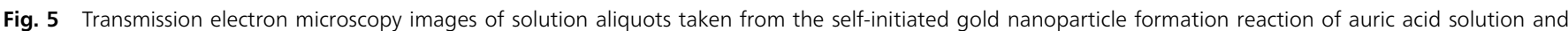

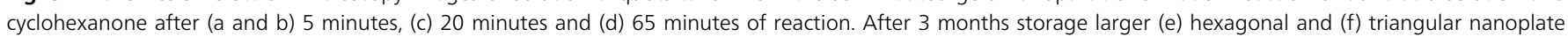
structures were also present.

The distribution of nanoparticle sizes and shapes are summarized in the histograms shown in Fig. 6. The histogram plots for the nanostructures observed after 20 and 65 minutes of reaction and 3 months storage were skewed distributions. The histogram after 5 minutes reaction was very much skewed to the left due to the large number of minute spherical particles that were present within the initial large dendritic structures. The overall average diameter of all nanostructures increased during the reaction period from $\approx 15 \mathrm{~nm}$ after 5 minutes, to $\approx 25 \mathrm{~nm}$ after 20 minutes, to $\approx 40 \mathrm{~nm}$ after 65 minutes of reaction. After 3 months in storage the average nanoparticle size did not increase greatly $(\approx 40 \mathrm{~nm}$ ) and signified that the nanoparticle formation reaction was primarily over after 65 minutes of reaction. Although a multitude of $3 \mathrm{D}$ shapes and flat nanoplate structures were observed, spherically shaped nanoparticles were present in majority (70\% presence on average) throughout the reaction. For the duration of the reaction it was observed that the SPR band centre shifted from 543 to $576 \mathrm{~nm}$ (Fig. 3), denoting an average increase in size of $\approx 34 \mathrm{~nm} .{ }^{36}$ From TEM studies, a similar size change was observed for the spherical particles that were present in majority, increasing in average size from $11 \pm 7 \mathrm{~nm}$ after 5 minutes of reaction to $43 \pm 7 \mathrm{~nm}$ after 65 minutes of reaction; a $32 \mathrm{~nm}$ average size increase, hence correlating well with the SPR shift.
A gold nanoparticle solution that had been allowed to react to completion ( $>65$ minutes) was freeze dried to form a powder for XRD analysis. The pattern, shown in Fig. 7, was phase equivalent to that of bulk gold showing primitive face-centred cubic (FCC) symmetry ( $F m \overline{3} m, a=4.0781 \AA$ A). ${ }^{37}$ Through fitting the pattern to a Le Bail refined model, a slightly larger unit cell was observed, where $a=4.1031 \AA$. This equated to an average unit cell expansion of $1.5 \%$ compared with bulk gold. In measuring the relative heights of each diffraction peak, slight preferred growth was observed in the (200) plane. Through applying the Scherrer equation to each diffraction peak, an average crystallite size of $14 \mathrm{~nm}$ with a standard deviation of $1.4 \mathrm{~nm}$ was observed. This indicates that the particles observed by TEM with average size ca. $40 \mathrm{~nm}$ are in fact on average composed of multiple crystal grains.

The freeze dried powder was also investigated using IR spectroscopy within a pressed $\mathrm{KBr}$ disc. Cyclohexanone solution was investigated as a standard for comparison. The presence of no organic compound other than cyclohexanone was observed in both spectra. This indicated that cyclohexanone not only acts as a reducing agent in the self-initiated formation of gold nanoparticles from auric acid, but also seems to play a surfactant-type role for stabilizing these particles. In addition, the carbonyl $\mathrm{C}=\mathrm{O}$ dimer bond stretch did not within error shift in 


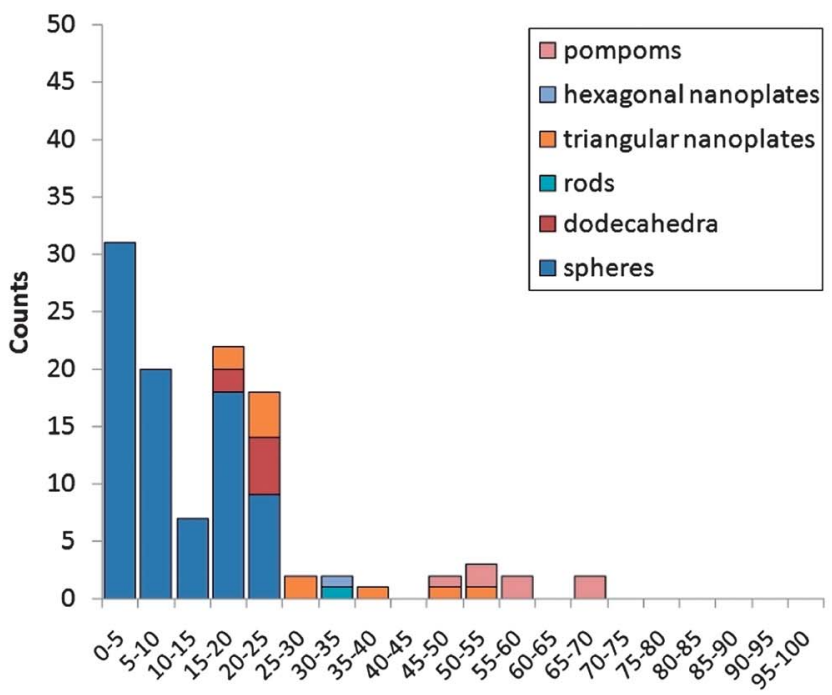

Particle Diameter (nm)

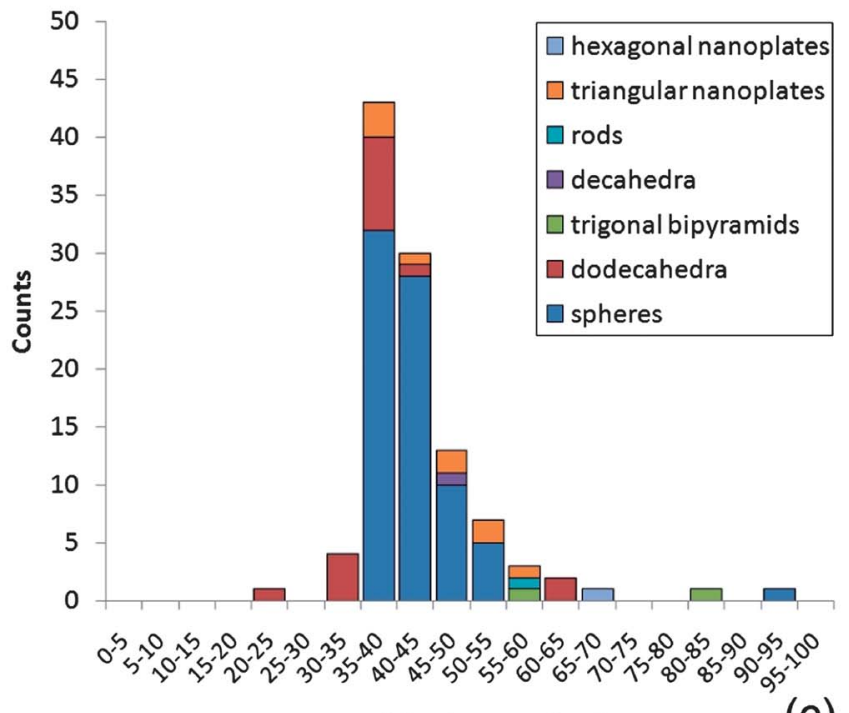

Particle Diameter (nm)

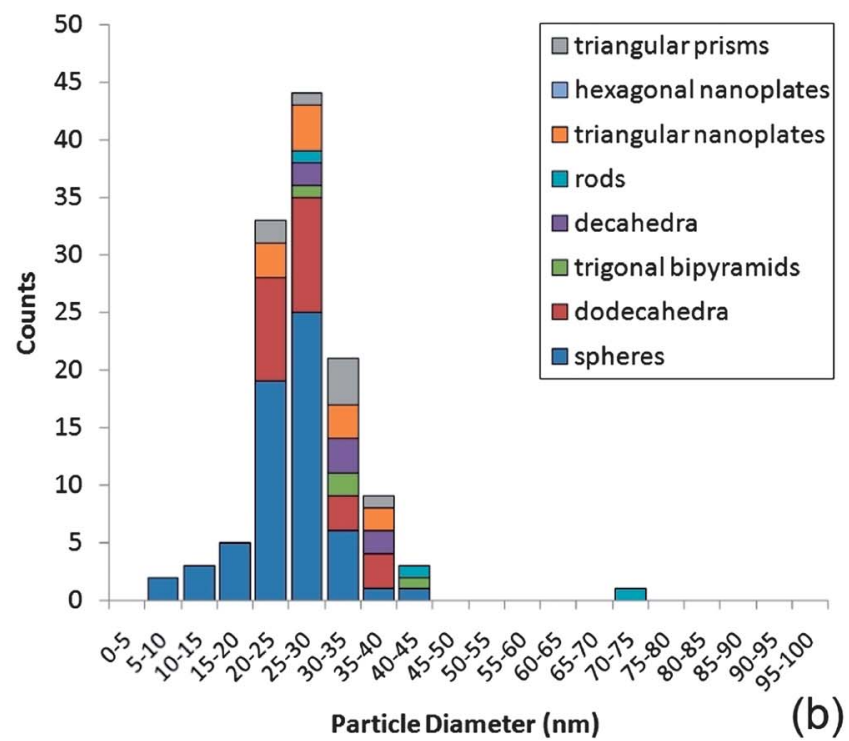

(b)

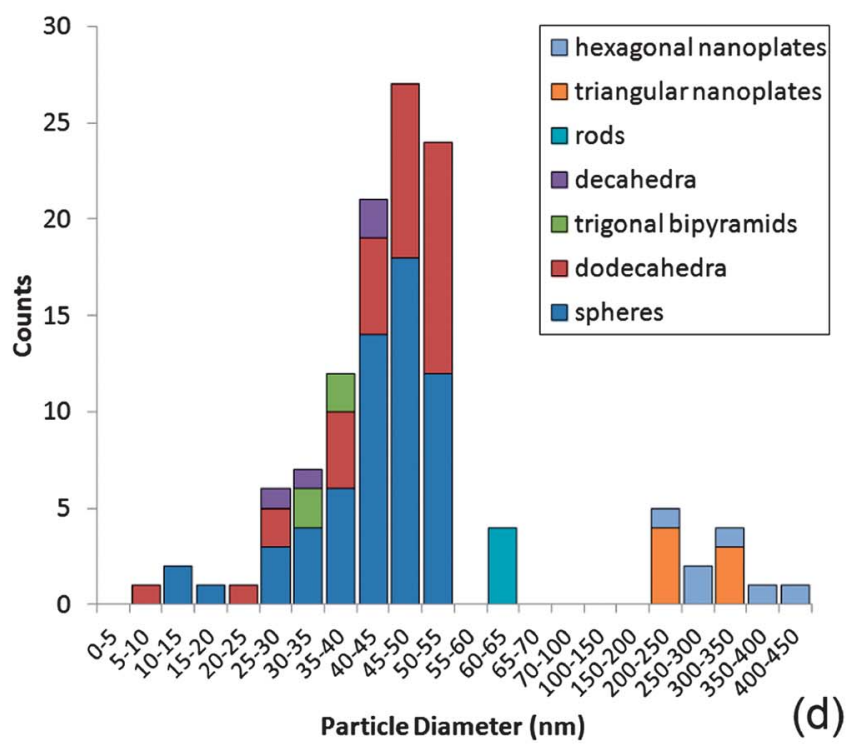

Fig. 6 Histogram plots of nanoparticle size ( $\mathrm{nm}$ ) and count for the various nanostructures observed after (a) 5 minutes, (b) 20 minutes and (c) 65 minutes of reaction from the self-initiated gold nanoparticle formation reaction of auric acid solution and cyclohexanone and (d) after 3 months storage.

the gold nanoparticle powder $\left(1714 \mathrm{~cm}^{-1}\right)$ compared with that of pure cyclohexanone $\left(1715 \mathrm{~cm}^{-1}\right)$. In Sarkosh et al.'s IR study of gold nanoparticles formed by ablating a gold target submerged in cyclohexanone solution an $\approx 24 \mathrm{~cm}^{-1}$ decrease in this $\mathrm{C}=\mathrm{O}$ stretch was observed and was attributed to the capping of cyclohexanone molecules to the gold nanoparticles through their $\mathrm{C}=\mathrm{O}$ bond at oxygen. In comparison, this indicates that either cyclohexanone did not preferentially bind through this functional group to gold in the colloid reported in this paper or that a number of cyclohexanone layers were present, stacking randomly around each nanoparticle.

\section{Mechanistic studies}

Gold nanoparticles were formed by simply mixing cyclohexanone with an aqueous solution of auric acid. The reaction took place at room temperature, unlike typical $\mathrm{Au}$ nanoparticle syntheses, without the need for any initiation i.e. thermal activation, sonication, photo-activation, etc. Three components were present in the solution; water, auric acid and cyclohexanone.

The reaction was repeated in $\mathrm{D}_{2} \mathrm{O}$ so that any by-products could be examined using ${ }^{1} \mathrm{H}$ and ${ }^{13} \mathrm{C}$ NMR. In the ${ }^{13} \mathrm{C}$ NMR spectrum, several peaks not relating to the presence of the excess reducing agent, cyclohexanone $\left[{ }^{13} \mathrm{C}\right.$ NMR $\left(600 \mathrm{MHz}, \mathrm{D}_{2} \mathrm{O}\right)$ $\delta(\mathrm{ppm})$ 25.1, 27.2, 41.8, 128.2, 209.2 [ESI $1 \dagger\}$ ], were observed. These peaks $\left[{ }^{13} \mathrm{C}\right.$ NMR $\left(600 \mathrm{MHz}, \mathrm{D}_{2} \mathrm{O}\right) \delta 22.7,23.9,33.8,36.8$, $39.2,41.2,65.2,67.0,95.2,210.2,216.3\{$ ESI $2 \dagger\}]$ well-matched a pure spectrum of 2-chlorocyclohexanone $\left[{ }^{13} \mathrm{C}\right.$ NMR $(600 \mathrm{MHz}$, $\left.\mathrm{D}_{2} \mathrm{O}\right) \delta(\mathrm{ppm}) 22.6,24.7,27.7,30.9,33.8,37.4,39.1,41.2,65.9$, 67.0, 94.9, 210.1, 216.0 \{ESI 3†\}].

The two missing peaks at $\delta(\mathrm{ppm}) 27.7$ and 30.9 of 2chlorocyclohexanone were masked by the large presence of 


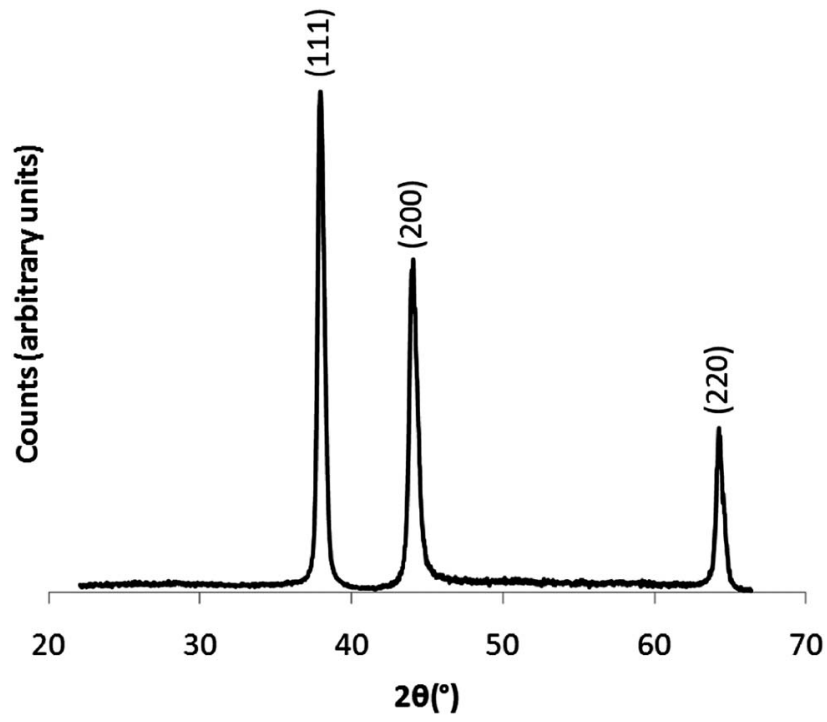

Fig. 7 X-ray diffraction pattern of a colloidal powder suspension. The powder was acquired through freeze-drying a gold nanoparticle solution, formed from the self-initiated reaction of auric acid solution and cyclohexanone that was allowed to react to completion (>65 minutes).

cyclohexanone in this region. Additional peaks were observed but not identified. A similar case was observed in ${ }^{1} \mathrm{H}$ NMR, where both cyclohexanone $\left[{ }^{1} \mathrm{H}\right.$ NMR $\left(600 \mathrm{MHz}, \mathrm{D}_{2} \mathrm{O}\right) \delta(\mathrm{ppm})$ 1.18 (2H, dddd), 1.36 (4H, dddd), 1.98 (4H, dd)] and 2-chlorocyclohexanone $\left[{ }^{1} \mathrm{H}\right.$ NMR $\left(600 \mathrm{MHz}, \mathrm{D}_{2} \mathrm{O}\right) \delta(\mathrm{ppm}) 1.38(1 \mathrm{H}, \mathrm{dtt})$, 1.51 (1H, dtt), $1.63(1 \mathrm{H}$, ddddd), $1.73(1 \mathrm{H}$, ddddd $), 1.82(1 \mathrm{H}$, dddd), 1.97 (1H, dddd), 2.57 (1H, ddd), 2.63 (1H, ddd), $4.05(1 \mathrm{H}$, dd)] environments were observed. The majority by-product was thus identified as 2-chlorocyclohexanone, which was shown to be identical to literature spectra and a dilute solution of the reagent. This was supported by gas chromatography measurements. Gas chromatography of the nanoparticle solution (containing the by-product) and clean measurements of pure 2chlorocyclohexanone was performed. Two matching peaks at the same retention time of 17.9 minutes were observed. Further confirmation of 2-chlorocyclohexanone being formed was found by IR spectroscopy. Measurements of the gas phase taken during the nanoparticle formation reaction in a gas-tight cell showed the progressive release of 2-chlorocyclohexanone (Fig. 7). A maximum concentration of 2-chlorocyclohexanone was reached after approximately 30 minutes, in line with titrations that indicated the complete reduction of auric acid in a similar time-frame (Fig. 8). Although cyclohexanone possesses a higher vapor pressure $\left(4.5 \mathrm{mmHg}, 25{ }^{\circ} \mathrm{C}\right)$ than 2-chlorocyclohexanone $\left(0.28 \mathrm{mmHg}, 25^{\circ} \mathrm{C}\right)$, the far poorer solubility of 2-chlorocyclohexanone in water $\left(0.02 \mathrm{~g} \mathrm{l}^{-1}\right)$ compared with cyclohexanone ( $87 \mathrm{~g} \mathrm{l}^{-1}$ ) was the over-riding factor that led to its existence in the gas phase. Assuming the $1: 1$ formation of 2chlorocyclohexanone from auric acid, $0.32 \mathrm{mg}$ would have formed during the reaction; $0.16 \mathrm{mg}$ over the solubility limit in $10 \mathrm{ml}$ of water and encouraging vaporization. On the other hand, when mixed thoroughly, cyclohexanone $(0.47 \mathrm{~g})$ was fully soluble, being $0.4 \mathrm{~g}$ under the solubility limit.

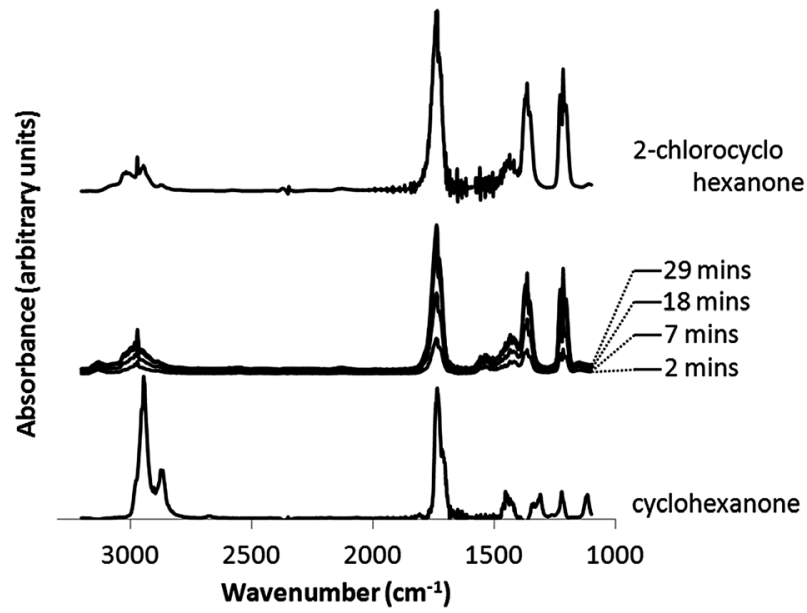

Fig. 8 IR spectra of the gas phase above the solution taken during the reaction of auric acid $(0.28 \mathrm{mM})$ with cyclohexanone $(0.48 \mathrm{M})$. Gas phase measurements of 2-chlorocyclohexanone and cyclohexanone are shown for reference.

The tautomerism of secondary ketones such as cyclohexanone is well known, forming an enol functional group with greater capacity for oxidation. ${ }^{38}$ The presence of this tautomer was indicated in this study as aqueous solutions of cyclohexanone discoloured bromine water (indicating an alkene group was present) and turned an acidified solution of $\mathrm{K}_{2} \mathrm{Cr}_{2} \mathrm{O}_{7}$ from orange to green upon slight heating (primary/secondary hydroxyl group present). In fact, a slight difference in the UVvisible absorption spectrum of aqueous cyclohexanone compared with pure was observed, when the peak at $913 \mathrm{~nm}$ in pure cyclohexanone disappeared in the aqueous mixture.

With the by-product and keto-enol chemistry in mind, a mechanism by which $\mathrm{Au}^{3+}$ could be reduced to $\mathrm{Au}^{0}$ is presented (Scheme 1). The mechanism was based in part on the similar chemistry observed between the pentavalent bismuth salt of $\mathrm{Ph}_{3} \mathrm{Bi}(\mathrm{v}) \mathrm{Cl}_{2}$ with cyclohexanone that leads to chlorine addition at the $\alpha$ position on cyclohexanone in a $2 \mathrm{e}^{-}$process forming 2 phenylcyclohexanone. ${ }^{39}$ Furthermore, Kochi demonstrated the reaction of $\mathrm{CuCl}_{2}$ with acetone in forming $\alpha$-chloro ketones, again a $2 \mathrm{e}^{-}$transfer process. ${ }^{40}$ To our knowledge, the comparable reaction of auric acid $\left(\mathrm{HAuCl}_{4}\right)$ and cyclohexanone has not been reported.

Auric acid can release $\mathrm{HCl}$ freely in solution, leaving $\mathrm{AuCl}_{3}$ (Scheme 1a). The acid can tautomerise cyclohexanone to the enol catalytically (Scheme $1 \mathrm{~b}$ ). Trigonal planar $\mathrm{AuCl}_{3}$ is then nucleophilically attacked by the lone pair on the enol leading to the $\mathrm{S}_{N} 2$ release of $\mathrm{Cl}^{-}$(Scheme 1c). The locality of the $\mathrm{Cl}-\mathrm{Au}-\mathrm{O}$ bond to the carbon $\alpha$ position induces a cyclic nucleophilic attack starting from the alkene bond. This leads to the reduction of gold from $\mathrm{Au}^{3+}\left(\mathrm{AuCl}_{3}\right)$ to $\mathrm{Au}^{1+}\left(\mathrm{AuCl}_{2}{ }^{-}\right)$and the formation of 2-chlorocyclohexanone.

As the reduction of a metal chloride by a ketone is a $2 \mathrm{e}^{-}$ transfer process, ${ }^{39,40}$ the direct reduction of $\mathrm{AuCl}_{2}{ }^{-}$to $\mathrm{Au}^{0}$ by cyclohexanone was not possible. Therefore, the disproportionation of $\mathrm{AuCl}_{2}{ }^{-}$to $\mathrm{Au}^{0}$ was the only viable route to $\mathrm{Au}^{0}$ :

$$
3 \mathrm{AuCl}_{2}^{-} \rightarrow 2 \mathrm{Au}^{0}+\mathrm{AuCl}_{4}^{-}+2 \mathrm{Cl}^{-}
$$


$\mathrm{HAuCl}_{4} \leftrightharpoons \mathrm{AuCl}_{3}+\mathrm{HCl}$

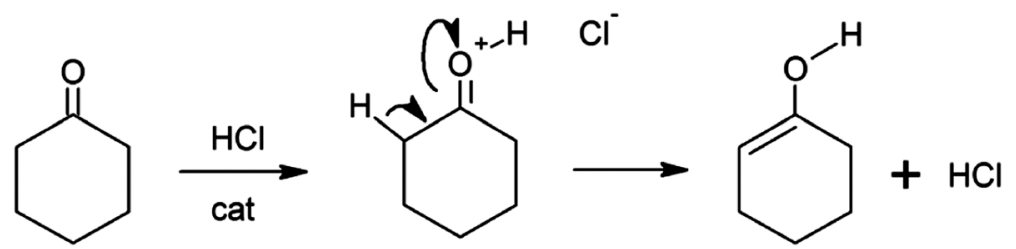

(1b)
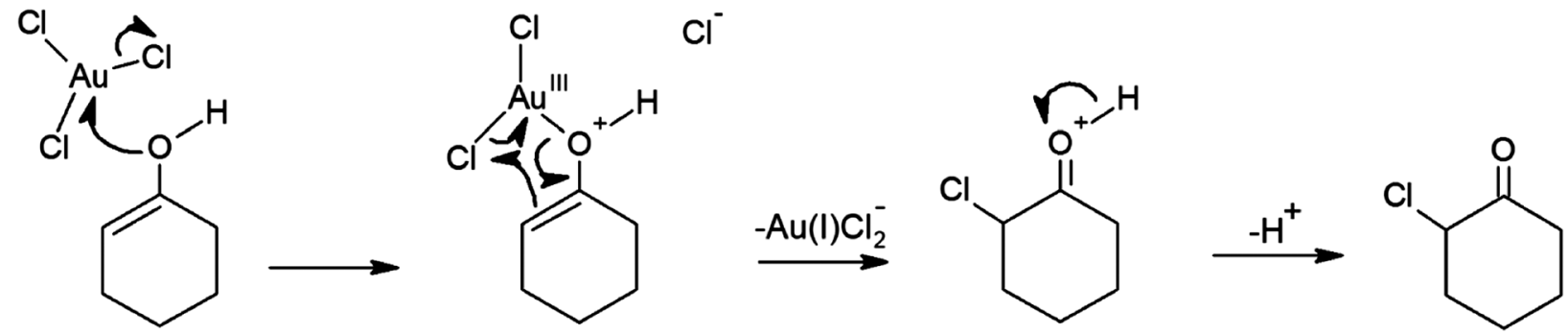

(1c)

Scheme 1 The mechanism for self-initiated gold nanoparticle growth from auric acid and cyclohexanone.

According to Gammons et al., the disproportionation of $\mathrm{AuCl}_{2}{ }^{-}$to $\mathrm{Au}^{0}$ takes place at room temperature. ${ }^{41}$ However, the rate of disproportionation increases rapidly with $\mathrm{AuCl}_{2}{ }^{-}$ concentration and temperature. Moreover, the reaction is catalyzed in the presence of $\mathrm{Au}$ foil. The fact that $\mathrm{Au}^{0}$ catalyzes the disproportionation of $\mathrm{AuCl}_{2}{ }^{-}$to $\mathrm{Au}^{0}$ shows that particle growth on $\mathrm{Au}^{0}$ seed sites will be favoured; thus leading to the nucleation and growth of nanoparticles through the continued absorption and disproportionation of $\mathrm{AuCl}_{2}{ }^{-}$. The steps can be summarized as follows:

$$
\begin{aligned}
3 \mathrm{C}_{5} \mathrm{H}_{10} \mathrm{CO}+3 \mathrm{HAuCl}_{4} \stackrel{-3 \mathrm{C}_{5} \mathrm{H}_{9} \mathrm{COCl}}{\longrightarrow} & 3 \mathrm{H}^{+}+3 \mathrm{HCl} \\
& +3 \mathrm{AuCl}_{2}{ }^{-} \rightarrow 2 \mathrm{Au}^{0}+\mathrm{HAuCl}_{4}+5 \mathrm{HCl}
\end{aligned}
$$

and the overall reaction as:

$$
3 \mathrm{C}_{5} \mathrm{H}_{10} \mathrm{CO}+2 \mathrm{HAuCl}_{4} \stackrel{-3 \mathrm{C}_{5} \mathrm{H}_{9} \mathrm{COCl}}{\longrightarrow} 2 \mathrm{Au}^{0}+5 \mathrm{HCl}
$$

Our TEM studies showed that large dendritic structures initially formed 5 minutes into the nanoparticle formation reaction. These structures then collapsed into smaller and denser particles with a variety of shapes. A similar growth mechanism has been observed in the Turkevich reaction (auric acid and tri-sodium citrate) between 70 and $100{ }^{\circ} \mathrm{C} .{ }^{31}$ Initially, large particles formed that were $\approx 100 \mathrm{~nm}$ in diameter that then collapsed over the course of the reaction into predominantly spherical shaped nanoparticles that were $\approx 20 \mathrm{~nm}$ in size. The similar nanoparticle growth mechanism observed in this study demonstrates the similar behaviour of the surfactants. Nevertheless, the observed phenomenon of particle shrinkage is akin to an inverse-Ostwald ripening process. ${ }^{42}$ This phenomenon was explained by Rodríguez-González et al. in terms of the specific adsorption of steric/electrosteric layers. ${ }^{32}$ Assessing the
Turkevich synthesis, Rodríguez-González et al. found that chloride ions primarily surround the large gold structures that initially form. However, when the $\mathrm{AuCl}_{4}{ }^{-}$is near consumed, the redox potential reduces allowing citrate ions to replace the surrounding chloride. This induces peptization as the gold surface is made more electrically neutral. We propose a similar phenomenon for the reaction of auric acid and cyclohexanone. We also suggest an extended Ostwald ripening ${ }^{43}$ process occurs over the 3 month storage period, causing the gold nanoplates to dramatically increase in size. We believe that the phenomenon is observed in the 2D shapes formed as opposed to the $3 \mathrm{D}$ shapes formed as growth in just a single plane facilitates significant increases in particle size.

\section{Conclusions}

A new and simple method for growing multi-shaped colloidal gold is presented. By adding cyclohexanone to aqueous solutions of auric acid, a self-initiating and self-propagating room temperature reaction occurs; resulting in the formation of gold nanoparticles. Such room temperature self-initiating nanoparticle synthesis reactions are different to traditional methods, such as the Turkevich method, where solutions require boiling temperatures to react. The formation proceeded in majority via large dendritic structures that collapsed to form more dense particles with a variety of shapes though most prominently spheres. The particles were highly crystalline and were stabilized by the cyclohexanone reducing agent, dually acting as a surfactant. Through identifying the 2-chlorocyclohexanone byproduct formed in this nanoparticle synthesis a mechanism by which $\mathrm{Au}^{3+}$ was reduced to $\mathrm{Au}^{0}$ was proposed. Furthermore, a new method of converting cyclic ketones to $\alpha$-chloro ketones was discovered. This new synthetic route to colloidal gold is straightforward and fast, forming nanoparticles with a range of 
shapes. The resulting nanoparticle solution is stable to precipitation for more than 3 months at room temperature. Surprisingly the flat nanoparticle sheets seem to grow over the 3 month period whereas the other nanoparticle shapes remain invariant after $c a .1 \mathrm{~h}$ of reaction.

\section{Acknowledgements}

IPP wishes to thank the EPSRC for funding. Dr Daniel Ortega is thanked for his help with TEM studies and Dr Abil Aliev is thanked for his help with NMR studies. Prof. William Motherwell and Dr Matthew Powner are thanked for help with the mechanism.

\section{References}

1 C. Burda, X. Chen, R. Narayanan and M. A. El-Sayed, Chem. Rev., 2005, 105, 1025.

2 G. Walters and I. P. Parkin, J. Mater. Chem., 2009, 19, 574.

3 K.-T. Yong, M. T. Swihart, H. Ding and P. N. Prasad, Plasmonics, 2009, 4, 79.

4 M. C. Ferrara, L. Mirenghi, A. Mevoli and L. Tapfer, Nanotechnology, 2008, 19, 365706.

5 R. G. Palgrave and I. P. Parkin, Chem. Mater., 2007, 19, 4639.

6 R. G. Palgrave and I. P. Parkin, J. Am. Chem. Soc., 2006, 128, 1587.

7 R. Binions, C. Piccirillo, R. G. Palgrave and I. P. Parkin, Chem. Vap. Deposition, 2008, 14, 33.

8 J. Gil-Tomas, S. Tubby, I. P. Parkin, N. Narband, L. Dekker, S. P. Nair, M. Wilson and C. Street, J. Mater. Chem., 2007, 17, 3739.

9 S. Perni, C. Piccirillo, J. Pratten, P. Prokopovich, W. Chrzanowski, I. P. Parkin and M. Wilson, Biomaterials, 2009, 30, 89.

10 S. I. Stoeva, J.-S. Lee, J. E. Smith, S. T. Rosen and C. A. Mirkin, J. Am. Chem. Soc., 2006, 128, 8378.

11 X.-M. Qian and S. M. Nie, Chem. Soc. Rev., 2008, 37, 912.

12 K.-S. Lee and M. A. El-Sayed, J. Phys. Chem. B, 2006, 110, 19220.

13 A. Corma and H. Garcia, Chem. Soc. Rev., 2008, 37, 2096.

14 J. Kimling, M. Maier, B. Okenve, V. Kotaidis, H. Ballot and A. Plech, J. Phys. Chem. B, 2006, 110, 15700.

15 J. Turkevich, P. C. Stevenson and J. Hillier, Discuss. Faraday Soc., 1951, 11, 55.

16 Y. Tai, N. T. T. Tran, Y.-C. Tsai, J.-Y. Fang and L.-W. Chang, IET Nanobiotechnol., 2011, 5, 52.

17 O. Bar-Ilan, R. M. Albrecht, V. E. Fako and D. Y. Furgeson, Small, 2009, 5, 1897.
18 B. D. Chithrani, A. A. Ghazani and W. C. W. Chan, Nano Lett., 2006, 6, 662.

19 Y. Ohko, T. Tatsuma, T. Fujii, K. Naoi, C. Niwa, Y. Kubota and A. Fujishima, Nat. Mater., 2003, 2, 29.

20 C. Huang, P. Chiu, Y. Wang, W. R. Chen and T. H. Meen, J. Electrochem. Soc., 2006, 153, D129.

21 C. Huang, Y. Wang, P. Chiu, M. Shih and T. H. Meen, Mater. Lett., 2006, 60, 1896.

22 X. Tong, Y. Zhao, T. Huang, H. Liu and K. Y. Liew, Appl. Surf. Sci., 2009, 255, 9463.

23 B. K. Jena and C. R. Raj, Chem. Mater., 2008, 20, 3546.

24 A. Ali Umar and M. Oyama, Cryst. Growth Des., 2009, 9, 1146.

25 C. Wang, C. Kan, J. Zhu, X. Zeng, X. Wang, H. Li and D. Shi, J. Nanomater., 2010, 2010, 1.

26 M. Tsuji, M. Hashimoto, Y. Nishizawa, M. Kubokawa and T. Tsuji, Chem.-Eur. J., 2005, 11, 440.

27 S. S. Shankar, A. Rai, B. Ankamwar, A. Singh, A. Ahmad and M. Sastry, Nat. Mater., 2004, 3, 482.

28 S. Biswas, S. K. Hait, S. C. Bhattacharya and S. P. Moulik, J. Dispersion Sci. Technol., 2005, 25, 801.

29 L. Sarkhosh, H. Aleali, R. Karimzadeh and N. Mansour, Phys. Status Solidi A, 2010, 207, 2303.

30 M. L. Marin, K. L. McGilvray and J. C. Scaiano, J. Am. Chem. Soc., 2008, 130, 16572.

31 M. K. Chow and C. F. Zukowski, J. Colloid Interface Sci., 1994, $165,97$.

32 B. Rodríguez-González, P. Mulvaney and L. M. Liz-Marzán, Z. Phys. Chem., 2007, 221, 415.

33 D. Ray, V. K. Aswal and J. Kohlbrecher, Langmuir, 2011, 27, 4048.

34 A. I. Yanson, P. Rodriguez, N. Garcia-Araez, R. V. Mom, F. D. Tichelaar and M. T. M. Koper, Angew. Chem., Int. Ed., 2011, 50, 6346.

35 V. Herschlog, Ind. Eng. Chem., Anal. Ed., 1941, 13, 561.

36 W. Haiss, N. T. K. Thanh, J. Aveyard and D. G. Fernig, Anal. Chem., 2007, 79, 4215.

37 E. A. Owen and E. L. Yates, J. Chem. Phys., 1935, 3, 605.

38 A. K. Cederstav and B. M. Novak, J. Am. Chem. Soc., 1994, 116, 4073.

39 D. H. R. Barton, J.-C. Blazejewski, B. Charpiot, J.-P. Finet, W. B. Motherwell, M. T. B. Papoula and S. P. Stanforth, J. Chem. Soc., Perkin Trans. 1, 1985, 2667.

40 J. K. Kochi, J. Am. Chem. Soc., 1955, 77, 5274.

41 C. H. Gammons, Y. Yu and A. E. Williams-Jones, Geochim. Cosmochim. Acta, 1997, 61, 1971.

42 D. Lee, S. Park, J. Lee and N. Hwang, Acta Mater., 2007, 55, 5281.

43 P. W. Voorhees, J. Stat. Phys., 1985, 38, 231. 